\title{
EL USO DE LA LEY DE TAYLOR EN EL ESTABLECIMIENTO DE PATRONES DE VARIACIÓN ESPACIO-TEMPORAL EN POBLACIONES ANIMALES: DOS EJEMPLOS DE APLICACIÓN
} Alfredo Giraldo Mendoza ${ }^{1}$, Claudia Véliz Rosas ${ }^{2}$, Germán Arellano Cruz y Edgard Sánchez Infantas ${ }^{2}$

\section{Resumen}

Se aplicó la Ley de Taylor en dos conjuntos de datos correspondientes a poblaciones de taxa distintos, con el propósito de determinar sus patrones espacio-temporales según la interpretación biológica de dicha ley y señalar sus limitaciones en la descripción de los cambios en la distribución espacial de las abundancias. Los datos empleados fueron las abundancias de aves entre Febrero-1998 y Febrero-2001 y, las abundancias de coleópteros colectados con trampas pitfall entre Febrero-1998 y Diciembre-2001 en la Reserva Nacional de Lachay. A través de la significación estadística de los valores de bs y bt, se estableció el patrón de refugios para 8 especies de aves y 5 de coleópteros, aunque los valores de $\mathrm{R}^{2}$ revelaron que éstos sólo se cumplieron parcialmente. Estos resultados indican que ninguna de las especies de ambos taxa se adecuó absolutamente a los patrones planteados como interpretación biológica de la Ley de Taylor y asimismo, que los valores de $\mathrm{R}^{2}$ deben tomarse en cuenta, como exigencia estadística necesaria para definir los patrones.

Palabras clave: Ley de Taylor, patrones de variación espacio-temporal, Aves, Coleoptera.

\begin{abstract}
The Taylor's power law was applied to two groups of data corresponding to populations of different taxa, with the purpose of determining their spatio-temporal patterns according to the biological interpretation of this law and to point out limitations in the description of the changes in the spatial distribution of the abundances. The data were the abundances of birds from February1998 to February-2001 and the abundances of beetles collected with pitfall traps from February1998 to December-2001 at the Lachay National Reserve. Statistical significance of the bs and bt values, established the refuge pattern for 8 bird species and for 5 beetle species, although the $\mathrm{R}^{2}$ values revealed that they complied only partially. These results indicate that none of the species of both taxa adjusted absolutely to the patterns outlined as the biological interpretation of the Taylor's power law and that the $\mathrm{R}^{2}$ values should be taken into account, as a necessary statistical demand to define the patterns.
\end{abstract}

Key words: Taylor's power law, patterns of spatio-temporal variation, Aves, Coleoptera.

\section{Introducción}

La Ley de Taylor ó ley de la media es la relación entre la variancia muestral $\left(\mathrm{S}^{2}\right)$ y la media muestral (m) de las densidades poblacionales, propuesta por Taylor (1961), expresada por la siguiente fómula:

$$
\mathrm{S}^{2}=\mathrm{a} \mathrm{m}
$$

De la cual se deduce la regresión:

$$
\log _{10} \mathrm{~S}^{2}=\log _{10} \mathrm{a}+\operatorname{blog}_{10} \mathrm{~m}
$$

Dicha relación fue destacada como el modelo más adecuado para la descripción de las distribuciones espaciales resultantes de muestreos poblacionales frente a los modelos de Iwao, de Poisson y binomial negativo (Taylor et al., 1978). Para ello emplearon 156 sets de datos correspondientes a los más variados taxa, métodos de muestreo y escalas espaciales. Estudios sucesivos (Taylor et al., 1980; Taylor \& Woiwod, 1982) harían énfasis en la universalidad del modelo y en su potencialidad para detectar comportamientos densodependientes en las dinámicas poblacionales. Cabe resaltar que a partir de Taylor \& Woiwod (1980, citado por Taylor \& Woiwod, 1982) se extendió la validez de la relación al ámbito temporal.

Posteriormente, Soberón \& Loevinsohn (1987) ofrecieron una serie de posibles interpretaciones biológicas a los valores de $\mathrm{b}=2, \mathrm{~b}<2$ y $\mathrm{b}>2$, obtenidos al aplicar la ley de Taylor, en datos obtenidos con distintos tipos de muestreo y de procesamiento de datos: muestreo espacial/ploteo temporal, muestreo espacial/ploteo espacial y muestreo temporal/ploteo espacial. Por su parte, Perry (1988) exploró los posibles mecanismos generadores de la ley de la

\footnotetext{
${ }^{1}$ Laboratorio de Control Biológico y Ecología de Artrópodos. Dpto. de Biología. Universidad Nacional Agraria La Molina. Av. La Universidad s/n. Apartado 456. Lima 100. E-mail: troodon76@hotmail.com acg@lamolina.edu.pe

${ }_{2}^{2}$ Laboratorio de Ecología de Procesos, Dpto. de Biología, Universidad Nacional Agraria La Molina Lima Av. La Universidad s/n. Apartado 456. Lima 100. E-mail: gaviotin@universia.edu.pe / esi@lamolina.edu.pe
} 
media, a través de la simulación con tres modelos poblacionales.

Mc Ardle et al. (1990) tomando como ejemplos de aplicación series de datos de áfidos y polillas previamente empleados (Taylor et al., 1980; Taylor \& Woiwod, 1982; Redfearn \& Pimm, 1988), propusieron el uso de la ley de Taylor como un método útil para detectar diferentes tipos de patrones de variabilidad espacio-temporal en poblaciones de una especie y, para efectuar comparaciones de variabilidad espacial y temporal entre poblaciones de especies diferentes. Con esta finalidad, dichos autores reemplazaron a la variancia por el coeficiente de variabilidad, como medida de variabilidad poblacional, dado que éste no se ve afectado por los ceros y permite la comparación entre especies. En consecuencia, la relación original fue cambiada por la siguiente expresión:

$$
\begin{gathered}
\text { c.v. }=a^{0.5} \mathrm{~m}^{(0.5 \mathrm{~b}-1)} \\
\log _{10} \text { c.v. }=0.5 \log _{10} \mathrm{a}+(0.5 \mathrm{~b}-1) \log _{10} \mathrm{~m}
\end{gathered}
$$

Los valores de b calculados con la expresión anterior, se convierten en descriptores del comportamiento de la variabilidad espacial (bs) o temporal (bt) de las abundancias medias de una población. Con base en las interpretaciones previas de Soberón \& Loevinsohn (1987), Mc. Ardle et al. (1990) propusieron las siguientes denominaciones, para los patrones espacio-temporales que emergen al combinar los valores de bs y bt: hot-spots $(\mathrm{bs}>2$, $\mathrm{b} t>2)$, refugios $(\mathrm{bs}<2, \mathrm{~b} t<2) \mathrm{y}$, hot-spots fijos $\mathrm{y}$ móviles ( $\mathrm{bs}>2, \mathrm{~b} \mathrm{t}<2)$.

El presente trabajo se propone aplicar la ley de taylor a las poblaciones de dos taxa evaluados simultáneamente, con el fin de determinar sus patrones espacio-temporales según la interpretación biológica de dicha ley y asimismo, señalar sus limitaciones en la descripción de los cambios en la distribución espacial de las abundancias.

\section{Materiales y métodos}

Se emplearon los datos de abundancia de aves registrados mediante conteo por puntos (Bibby et al., 1993) en 31 estaciones distribuidas en un sector de aproximadamente 430 ha. en la Reserva Nacional de Lachay $\left(11^{\circ} 21^{\prime} 00^{\prime \prime}-11^{\circ} 21^{\prime} 58^{\prime \prime}\right.$ L.S. y $77^{\circ} 22^{\prime} 25^{\prime \prime}$ $77^{\circ} 22^{\prime} 28^{\prime \prime}$ L.O.) durante 13 muestreos efectuados entre Febrero-1998 y Febrero-2001 (Véliz, 2002). Los datos de abundancia de los coleópteros fueron obtenidos por medio de trampas pitfall ubicadas en las mismas estaciones empledaas para las aves, durante 15 muestreos efectuados entre Febrero-1998 y Diciembre-2001 (Giraldo, 2002). El período de estudio estuvo influenciado por el evento El Niño 1997-98, el cual incrementó el volumen de precipitaciones por encima de sus niveles normales durante 1998 (Figura 1).

Los análisis se realizaron sólo con las especies más abundantes de ambos taxa, 18 de aves y 20 de coleópteros. Las regresiones entre $\operatorname{los} \log _{10}$ c.v. y los $\log _{10} \mathrm{~m}$ de las abundancias, fueron realizadas con Statistica versión 6.0, para obtener los valores de los parámetros a y $b$, el coeficiente de determinación $\mathrm{R}^{2}$, así como el error estándar de b.

Para comprobar que los valores de b obtenidos fueran significativamente distintos de 2 se emplearon dos métodos:

(1) Cálculo de intervalos de confianza usando la distribución $\mathrm{t}$, con un $\mathrm{a}=0.05$ y g.l. $=\mathrm{n}-2$, según la siguiente fórmula (Steel y Torrie, 1988):

$$
\text { I.C.(b) }=\mathrm{b} \pm \mathrm{t}_{\mathrm{a} / 2} \cdot \mathrm{S}_{\mathrm{b}}^{2}
$$

Donde: $b=$ pendiente de la recta

$\mathrm{t}_{\mathrm{a} / 2}=$ valor tabular de la distribución $\mathrm{t}$

$\mathrm{S}_{\mathrm{b}}{ }^{2}=$ error estándar de la pendiente

(2) Prueba F para contrastar la hipótesis nula Ho: b $=2$, con un nivel de significación de $\mathrm{a}=0.05$.

Asimismo, para tener un término de referencia, se generaron patrones de refugios "ideales", alterando el orden de los datos pertenecientes a las cinco especies de Coleoptera cuyos valores de bs y bt fueron significativamente menores a 2 , de manera que los mayores valores de abundancia siempre se presentaran en las mismas estaciones de muestreo.

\section{Resultados y discusión}

De las especies que fueron seleccionadas, sólo 8 aves y 5 coleópteros presentaron ambos valores de $\mathrm{b}$ significativamente distintos a 2, éstas se presentan en la Tabla $n^{\circ} 1$. En todos los casos, los valores de $b$ fueron menores a 2, por lo cual les correspondería el patrón de refugios, es decir sus poblaciones serían menos variables a través del tiempo en los hábitats favorables que en los desfavorables y durante las malas temporadas tales poblaciones tenderían a refugiarse sólo en algunos sitios (aquellos más favorables) incrementando así la variabilidad espacial.

Al examinar los refugios "ideales" (Tabla $n^{\circ} 2$ ) se observa que éstos presentan valores de $\mathrm{R}^{2}$ superiores a 0.6 en todas sus regresiones. En contraste, los refugios obtenidos con datos reales presentan en su mayoría altos valores de $\mathrm{R}^{2}(>0.6)$ en las regresiones realizadas para obtener el bs, pero bajos valores de $\mathrm{R}^{2}$ en las regresiones realizadas para obtener el bt $(<\mathrm{o}=$ $0.6)$. Las excepciones a esta tendencia general son Pyrocephalus rubinus, que muestra un comportamiento inverso $\left(\mathrm{R}^{2}\right.$ espacial $<0.6 \mathrm{y} \mathrm{R}^{2}$ temporal > 0.6), Sturnella bellicosa, que tiene ambos valores de $\mathrm{R}^{2}$ bajos y Carduelis magellanicus que resulta ser la única especie con ambos valores de $\mathrm{R}^{2}$ 
mayores a 0.6. Sin embargo, para éste último caso hay que notar que se cuentan con muy pocos grados de libertad para la variabilidad espacial $(\mathrm{g} .1 .=2, \mathrm{n}=4)$, lo cual hace del resultado susceptible a variaciones posteriores si se incrementara el muestreo.

Resulta entonces que si bien los valores de b por sí solos nos indicarían la predominancia del patrón de refugios, los valores de $\mathrm{R}^{2}$ evidencian que éste sólo se cumple parcialmente, de modo que la variabilidad espacial cambió notoriamente y de manera definida a través del tiempo, pero sin establecer distinciones claras entre hábitats favorables y desfavorables. Sólo en el caso de Pyrocephalus rubinus y Carduelis magellanicus hubo una clara preferencia de hábitat a tráves del tiempo. Por su parte, Sturnella bellicosa, no manifestó una selección clara por hábitats ó temporadas. El escrutinio de las distribuciones espaciales de las abundancias mediante el programa Surfer 7.0, corrobora estas afirmaciones (Giraldo, 2002; Véliz, com. pers.).

En vista de lo expuesto anteriormente, es probable que para la mayoría de especies de ambos taxa, la estacionalidad y la perturbación de El Niño 1997-98, operando en el ámbito temporal, tuvieron una mayor importancia en la determinación de los patrones de variación poblacional, que cualquier heterogeneidad espacial presente en el área de estudio.

La importancia de definir con la máxima precisión posible la existencia y la ubicación de refugios deja de ser una preocupación meramente teórica, si tomamos en cuenta que su identificación sería crucial para establecer zonificaciones que permitan la conservación ó la explotación sostenida de una población. Pulliam (1988) manifestó una preocupación similar, con respecto a las decisiones erróneas que podrían tomarse en materia de manejo poblacional, si los estudios que respaldan tales decisiones no lograsen determinar la ubicación de las fuentes (modelo metapoblacional fuente-sumidero).

\section{Conclusiones}

- Ninguna de las especies de ambos taxa se adecuó absolutamente a los patrones planteados como interpretación biológica de la Ley de Taylor.

- El valor del coeficiente de determinación $\left(\mathrm{R}^{2}\right)$ debe ser considerado como requisito estadístico necesario para la definición de los patrones, además de la significación de los valores de b.

\section{Agradecimientos}

Los autores agradecen al Consejo Nacional de Ciencia y Tecnología (CONCYTEC), por el apoyo financiero para la realización de las salidas de campo. Al personal de la Reserva Nacional de Lachay por las facilidades brindadas durante cada uno de los muestreos.

\section{Literatura citada}

Bibby C., Burgess N. \& Hill D. 1993. Bird census techniques. Academic Press. Harcourt Brace \& company publishers. London.

Giraldo A. 2002. Análisis de los patrones de variación espacio-temporal de las poblaciones de coleópteros en la Reserva Nacional de Lachay durante el periodo 1998 - 2001. Tesis para optar el Título de Biólogo - Universidad Nacional Agraria La Molina. Lima Perú.

Mc. Ardle B.H., Gaston K.J. \& Lawton J.H. 1990. Variation in the size of animal populations: patterns, problems and artefacts. Journal of Animal Ecology. 59: 439-454.

Perry J.N. 1988. Some models for spatial variability of animal species. Oikos. 51: 124-130.

Pulliam H.R. 1988. Sources, sinks and population regulation. The American Naturalist. 132: 652 661.

Redfearn A. \& Pimm S. 1988. Population variability and polyphagy in herbivorous insect communities. Ecological Monographs. 58(1): 39-55.

Soberón J. \& Loevinsohn M. 1987. Patterns of variations in the numbers of animal populations and the biological foundations of Taylor's law of the mean. Oikos. 48: 249-252.

Steel R. y Torrie J. 1988. Bioestadística: Principios y procedimientos. Mc Graw-Hill / Interamericana de México S.A. de C.V.

Taylor L.R. 1961. Aggregation, variance and the mean. Nature. 189: $732-735$.

Taylor L.R. \& Woiwod I.P. 1982. Comparative synoptic dynamics I. Relationships between inter and intra-specific spatial and temporal variance/mean populations parameters. Journal of Animal Ecology. 51: 879-906.

Taylor L.R., Woiwod I.P. \& Perry J.N. 1978. The density-dependence of spatial behaviour and the rarity of randomness. Journal of Animal Ecology. 47: 383-406.

Taylor L.R., Woiwod I.P. \& Perry J.N. 1980. Variance and the large scale spatial stability of aphids, moths and birds. Journal of Animal Ecology. 49: 831-854.

Véliz C. 2002. Resiliencia de comunidades de aves en la Reserva Nacional de Lachay luego del evento El Niño 1997 - 98. Tesis para optar el Título de Bióloga-UNALM. 
Tabla $n^{\circ} 1$. Valores de $b$, intervalos de confianza y coeficientes de determinación $\mathrm{R}^{2}$ para las especies de aves y de coleópteros con b significativamente distintos de 2

\begin{tabular}{|c|c|c|c|c|c|c|}
\hline & bs & I.C. & $\mathrm{R}^{2}$ & bt & I.C. & $\mathbf{R}^{2}$ \\
\hline \multicolumn{7}{|l|}{ Aves } \\
\hline Bolborhyncus aurifrons & 1,47 & $1.3-1.64$ & 0,81 & 1,69 & $1.53-1.85$ & 0,36 \\
\hline Carduelis magellanicus & 1,21 & $0.88-1.53$ & 0,96 & 1,7 & $1.54-1.85$ & 0,61 \\
\hline Conirostrum cinereum & 1,35 & $1.01-1.7$ & 0,69 & 1,62 & $1.44-1.8$ & 0,32 \\
\hline Poospiza hispaniolensis & 1,29 & $0.94-1.64$ & 0,64 & 1,38 & $1.14-1.63$ & 0,49 \\
\hline Pyrocephalus rubinus (1) & 1,17 & $0.66-1.68$ & 0,44 & 1,12 & $1.01-1.23$ & 0,86 \\
\hline Sturnella belicosa (2) & 1,67 & $1.29-1.78$ & 0,40 & 1,59 & $1.44-1.75$ & 0,44 \\
\hline Troglodytes aedon & 1,13 & $0.84-1.43$ & 0,72 & 1,31 & $1.11-1.52$ & 0,55 \\
\hline Volatinia jacarina & 1,23 & $1.03-1.5$ & 0,88 & 1,71 & $1.55-1.86$ & 0,31 \\
\hline \multicolumn{7}{|l|}{ Coleoptera } \\
\hline Calosoma rufipenne & 1,43 & $1.36-1.50$ & 0,91 & 1,83 & $1.76-1.89$ & 0,20 \\
\hline Clambus sp. & 1,42 & $1.34-1.49$ & 0,86 & 1,62 & $1.55-1.69$ & 0,48 \\
\hline Epitrix sp. & 1,52 & $1.42-1.63$ & 0,73 & 1,70 & $1.58-1.82$ & 0,20 \\
\hline Tropiphorini-1 & 1,55 & $1.44-1.67$ & 0,70 & 1,64 & $1.56-1.73$ & 0,56 \\
\hline Tropiphorini-2 & 1,59 & $1.48-1.70$ & 0,62 & 1,61 & $1.50-1.71$ & 0,39 \\
\hline
\end{tabular}

(1) El valor de $R^{2}$ es mayor en la regresión de bt

(2) Ambos valores de $R^{2}$ son bajos $(<0,6)$

Tabla $\mathbf{n}^{\circ} \mathbf{2}$. Valores de $\mathrm{b}$, intervalos de confianza y coeficientes de regresión $\mathrm{R}^{2}$ para los refugios obtenidos a partir de los datos modificados de coleoptera

\begin{tabular}{|ccc|ccc|}
\hline bs & I.C. & $\mathbf{R}^{2}$ & bt & I.C. & $\mathbf{R}^{2}$ \\
\hline
\end{tabular}

Coleoptera (modificado)

\begin{tabular}{|c|c|c|c|c|c|c|}
\hline Calosoma rufipenne & 1,43 & $1.36-1.50$ & 0,91 & 1,73 & $1,69-1,76$ & 0,78 \\
\hline Clambus sp. & 1,42 & $1.34-1.49$ & 0,86 & 1,49 & $1,45-1,52$ & 0,93 \\
\hline Epitrix sp. & 1,52 & $1.42-1.63$ & 0,73 & 1,42 & $1,37-1,48$ & 0,93 \\
\hline Troiphorini-1 & 1,55 & $1.44-1.67$ & 0.70 & 1,74 & $1,68-1,79$ & 0,86 \\
\hline Troiphorini-2 & 1,59 & $1.48-1.70$ & 0,62 & 1,24 & $1,16-1,32$ & 0,93 \\
\hline
\end{tabular}

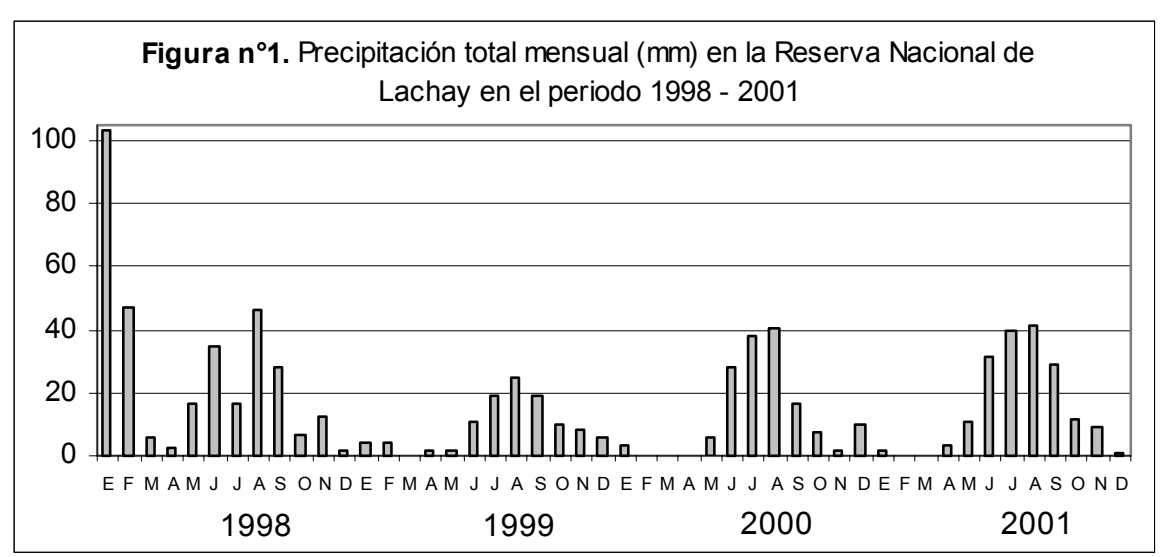

Datos proporcionados por la estación meteorológica de la Reserva 